\title{
On local quasi efficient solutions for nonsmooth vector optimization problems
}

\author{
Mohsine Jennane ${ }^{1}$, Lhoussain El Fadil ${ }^{1}$ and El Mostafa Kalmoun ${ }^{2, *}$ \\ ${ }^{1}$ Faculty of Sciences Dhar El Mahraz, Sidi Mohammed Ben Abdellah University \\ P.O. Box 1796 Atlas, 30050 Fes, Morocco \\ E-mail: 〈mohsine.jennane@usmba.ac.ma〉,〈lhouelfadil2@gmail.com〉 \\ ${ }^{2}$ Department of Mathematics, Statistics and Physics \\ Qatar University, Doha, Qatar \\ E-mail: 〈ekalmoun@qu.edu.qa〉
}

\begin{abstract}
We are interested in local quasi efficient solutions for nonsmooth vector optimization problems under new generalized approximate invexity assumptions. We formulate necessary and sufficient optimality conditions based on Stampacchia and Minty types of vector variational inequalities involving Clarke's generalized Jacobians. We also establish the relationship between local quasi weak efficient solutions and vector critical points.
\end{abstract}

Keywords: generalized approximate invexity, nonsmooth vector optimization, quasi efficient solutions, vector variational inequalities

Received: December 04, 2019; accepted: April 02, 2020; available online: July 07, 2020

DOI: $10.17535 /$ crorr.2020.0001

\section{Introduction}

Throughout the paper, $\mathbb{R}^{n}$ and $\mathbb{R}^{m}$ are the $n$-dimensional and the $m$-dimensional Euclidean spaces respectively, $X \subseteq \mathbb{R}^{n}$ is a nonempty open set and $f:=\left(f_{1}, \ldots, f_{m}\right): X \rightarrow \mathbb{R}^{m}$ is a vectorvalued function. Assume $C \subseteq \mathbb{R}^{m}$ is a closed pointed convex cone with a nonempty interior. Partial ordering is defined on $\mathbb{R}^{m}$ using $C$ as follows: $x \geq_{C} y$ (resp. $x>_{C} y$ ) if $x-y \in C$ (resp. $x-y \in \operatorname{int} C$ ) for all $x, y \in \mathbb{R}^{m}$.

Let us consider the following vector optimization problem (VOP)

$$
\min _{x \in X} f(x) .
$$

Recall that $\xi \in X$ is an efficient solution of (VOP), if no other feasible vector $x \in X$ satisfies $f(x) \leq_{C} f(\xi)$. Suppose we are given a mapping $\eta: X \times X \rightarrow \mathbb{R}^{n}$ and a vector $e \in \mathbb{R}^{m}$ such that $e>_{C} 0$. In this paper, we are interested in optimality conditions for a weaker type of solutions to vector optimization problem, i.e. local $(\eta, e)$-quasi efficient solutions.

Definition 1. We say that a feasible point $\xi \in X$ is a local ( $\eta, e)$-quasi (weak) efficient solution of (VOP) if there is $r>0$ such that there is no $x \in B(\xi ; r)$ satisfying $f(x)+e\|\eta(x, \xi)\| \leq_{C}$ $\left(<_{C}\right) f(\xi)$.

*Corresponding author. 
Vector optimization problems have a number of important applications in applied science, engineering and economics; see for example [9] and references therein. A powerful tool to study their optimality conditions is through vector variational inequalities [2], initiated by Giannessi [12] to be vector extensions of Stampacchia variational inequalities [22]. For differentiable and convex multiobjective functions, Giannessi [13] used vector variational inequalities of Minty type [17] to derive necessary and sufficient conditions for efficient solutions. Generalizations of these optimality conditions were given for different types of generalized convexity $[1,10,11,21$, $23]$ and generalized invexity [3, 16, 18, 24]. In [19], relationships between quasi efficient points, solutions to Stampacchia vector variational inequalities and vector critical points were identified under approximate convexity assumptions. On the other hand, a new concept of approximate invexity was defined in [4] as an extension of approximate convexity [20]. Furthermore, four new classes of generalized convexity were introduced in [7] as a generalization of the classical notions of pseudoconvexity and quasiconvexity.

It is worth mentioning that in case of nonsmooth vector optimization, the appropriate tool to study optimality conditions is Clarke's generalized Jacobian [8] when the multiobjective function is supposed to be locally Lipschitz. Recall that $f$ is locally Lipschitz if for any $x_{0} \in X$ there are two positive reals $k$ and $r>0$ with

$$
\|f(x)-f(y)\| \leq k\|x-y\|, \quad \forall x, y \in B\left(x_{0}, r\right) .
$$

In this case, Clarke's generalized Jacobian [8] of $f$ at $x \in X$ is the set of $m \times n$ matrices defined by

$$
\partial f(x)=c o\left\{\lim _{i \rightarrow+\infty} J f\left(x^{(i)}\right): x^{(i)} \rightarrow x, x^{(i)} \in S\right\}
$$

where co indicates the convex hull, $J f\left(x^{(i)}\right)$ is the Jacobian of $f$ at $x^{(i)}$, and $S$ is the differentiability set of $f$.

In many previous works $[14,15,18,19]$ Clarke's generalized Jacobian of $f$ at $x$ was defined to be the Cartesian product of its real-valued components Clarke's subdifferentials $\partial f_{1}(x) \times \ldots \times$ $\partial f_{m}(x)$. Using (1) in vector optimizations problems seems to be a more natural extension of the real-valued case since $\partial f$ as defined above is not equal to this Cartesian product. Nevertheless, the inclusion

$$
\partial f(x) \subseteq \partial f_{1}(x) \times \ldots \times \partial f_{m}(x)
$$

reveals that using $\partial f$ in the generalized convexity/invexity definitions and in the vector variational inequalities appear to be less restrictive than the Cartesian product.

Our aim in this paper is to introduce new types of generalized approximate invexity and investigate their use in deriving optimality conditions for local $(\eta, e)$-quasi (weak) efficient solutions of (VOP). In particular, we use both strong and weak forms of Stampacchia [22] and Minty [17] vector variational inequalities given in terms of Clarke's generalized Jacobian (1). Finally, we show also the relationship between local $(\eta, e)$-quasi weak efficient solutions and vector critical points.

\section{Generalized approximate invexity}

From now onward, we suppose that $f$ is locally Lipschitz. Let us present our extensions of the generalized approximate convexity concepts provided in $[5,7,20]$.

Definition 2. $f$ is said to be approximate $(\eta, e)$-invex at $x_{0} \in X$ if there is $r>0$ such that for any $x, y \in B\left(x_{0}, r\right)$,

$$
f(x)-f(y) \geq_{C} A_{y} \eta(x, y)-e\|\eta(x, y)\|, \forall A_{y} \in \partial f(y) .
$$

$f$ is said to be approximate $(\eta, e)$-invex on $X$, if $f$ is approximate $(\eta, e)$-invex at each $x_{0} \in X$. 
By taking $\eta(x, y)=x-y$, we deduce that approximate $e$-convexity [20], that is where (2) is replaced by

$$
f(x)-f(y) \geq_{C} A_{y}(x-y)-e\|x-y\|, \forall A_{y} \in \partial f(y),
$$

is a special case of approximate $(\eta, e)$-invexity. However, the following example shows that the class of approximate $(\eta, e)$-invex functions is strictly large than that of approximate $e$-convex ones.

Example 1. Let $X=\mathbb{R}, C=\mathbb{R}_{+}^{2}$ and for $x, y \in \mathbb{R}$

$$
f(x)=(x, \varphi(x))^{T} \quad \text { where } \quad \varphi(x)= \begin{cases}4 x-x^{2}, & x \geq 0 \\ 2 x, & x<0\end{cases}
$$

and

$$
\eta(x, y)=-|x-y|
$$

Clarke's generalized Jacobian of $f$ at $x$ is given by

$$
\partial f(x)= \begin{cases}\left\{(1,4-2 x)^{T}\right\}, & x>0 \\ \left\{(1, k)^{T}: k \in[2,4]\right\}, & x=0 \\ \left\{(1,2)^{T}\right\}, & x<0 .\end{cases}
$$

Let $x_{0}=0, e=(\varepsilon, \varepsilon)$ for an arbitrary real $\varepsilon>0$, and take $r=\min \left(1, \frac{\varepsilon}{2}\right)>0$. For all $x, y \in B\left(x_{0}, r\right)$ and all $A_{y} \in \partial f(y)$, we have

$$
f(x)-f(y)=(x-y, \varphi(x)-\varphi(y))^{T},
$$

where

$$
\varphi(x)-\varphi(y)= \begin{cases}(x-y)(4-x-y), & \text { if } y>0, x>0 ; \\ 2 x-4 y+y^{2}, & \text { if } y>0, x \leq 0 ; \\ 4 x-x^{2}-2 y, & \text { if } y<0, x \geq 0 \\ 2(x-y), & \text { if } y<0, x<0 \\ 4 x-x^{2}, & \text { if } y=0, x>0 ; \\ 2 x, & \text { if } y=0, x<0 ;\end{cases}
$$

and

$$
A_{y} \eta(x, y)-e\|\eta(x, y)\|=((-1-\varepsilon)|x-y|, \alpha(x, y))^{T},
$$

where

$$
\alpha(x, y)= \begin{cases}|x-y|(2 y-4-\varepsilon), & \text { if } y>0 \\ |x-y|(-2-\varepsilon), & \text { if } y<0 \\ |x|(-k-\varepsilon), & \text { if } y=0 .\end{cases}
$$

We can easily verify that $f(x)-f(y) \geq_{C} A_{y} \eta(x, y)-e\|\eta(x, y)\|$. Hence $f$ is approximate $(\eta, e)$ invex at $x_{0}=0$.

However, $f$ is not approximate convex. Indeed, if we take $y<0$ and $x=0$, then for $0<\varepsilon<1$ the inequality of approximate convexity is not satisfied.

Definition 3. Let $x_{0} \in X$. The function $f$ is said to be

- approximate pseudo $(\eta, e)$-invex of type $I$ at $x_{0}$ if there exists $r>0$ so that for any $x, y \in B\left(x_{0}, r\right)$,

$$
f(x)-f(y)<_{C}-e\|\eta(x, y)\| \quad \Rightarrow \quad A_{y} \eta(x, y)<_{C} 0, \forall A_{y} \in \partial f(y) ;
$$


- approximate pseudo $(\eta, e)$-invex of type II at $x_{0}$ if there exists $r>0$ so that for any $x, y \in B\left(x_{0}, r\right)$,

$$
f(x)-f(y)<_{C} 0 \quad \Rightarrow \quad A_{y} \eta(x, y)+e\|\eta(x, y)\|<_{C} 0, \forall A_{y} \in \partial f(y) .
$$

- approximate quasi $(\eta, e)$-invex of type $I$ at $x_{0}$ if there exists $r>0$ so that for any $x, y \in$ $B\left(x_{0}, r\right)$,

$$
\exists A_{y} \in \partial f(y): A_{y} \eta(x, y)-e\|\eta(x, y)\|>_{C} 0 \Rightarrow f(x)-f(y)>_{C} 0 .
$$

- approximate quasi $(\eta, e)$-invex of type II at $x_{0}$ if there exists $r>0$ so that for any $x, y \in B\left(x_{0}, r\right)$,

$$
\exists A_{y} \in \partial f(y): A_{y} \eta(x, y)>_{C} 0 \Rightarrow f(x)>_{C} f(y)+e\|\eta(x, y)\| .
$$

\section{Remark 1.}

- If $f$ is approximate pseudo (resp. quasi) $(\eta, e)$-invex of type II at $x_{0} \in X$, then $f$ is approximate pseudo (resp. quasi) $(\eta, e)$-invex of type I at $x_{0}$.

- It is easy to see that any approximate $(\eta, e)$-invex function at $x_{0}$ is approximate pseudo $(\eta, e)$-invex function of type I and approximate quasi $(\eta, e)$-invex function of type I at $x_{0}$.

- There is no relation between approximate pseudo $(\eta, e)$-invex functions of type II and approximate quasi $(\eta, e)$-invex functions of type II and approximate invex functions (see [14]).

\section{Sufficient conditions for local quasi efficient solutions}

We first consider Stampacchia and Minty types of vector variational inequalities involving Clarke's generalized Jacobians:

(SVVI) Find $\xi \in X$ for which there exists no $x \in X$ satisfying

$$
A_{\xi} \eta(x, \xi) \leq_{C} 0, \quad \forall A_{\xi} \in \partial f(\xi) .
$$

(MVVI) Find $\xi \in X$ for which there exists no $x \in X$ satisfying

$$
A_{x} \eta(x, \xi) \leq_{C} 0, \quad \forall A_{x} \in \partial f(x) .
$$

We present sufficient conditions for local $(\eta, e)$-quasi efficient solutions of (VOP) under approximate invexity assumptions.

Theorem 1. Suppose $f$ is approximate $(\eta, e)$-invex at $\xi \in X$. If $\xi$ is a solution of (SVVI), then $\xi$ is also a local $(\eta, e)$-quasi efficient solution of (VOP).

Proof. Assume $\xi$ fails to be a local $(\eta, e)$-quasi efficient solution of (VOP). Hence for each $r>0$ there is $x_{0} \in B(\xi, r)$ satisfying

$$
f\left(x_{0}\right)-f(\xi) \leq_{C}-e\left\|\eta\left(x_{0}, \xi\right)\right\| .
$$

Since $f$ is approximate $(\eta, e)$-invex at $\xi$, it follows that

$$
f\left(x_{0}\right)-f(\xi) \geq_{C} A_{\xi} \eta\left(x_{0}, \xi\right)-e\left\|\eta\left(x_{0}, \xi\right)\right\|, \quad \forall A_{\xi} \in \partial f(\xi) .
$$

Using (3), we get

$$
A_{\xi} \eta\left(x_{0}, \xi\right) \leq_{C} 0, \quad \forall A_{\xi} \in \partial f(\xi) .
$$

This means $\xi$ does not solve (SVVI). 
Remark 2. As the approximate invexity assumption is more general than approximate convexity, Theorem (1) extends Theorem 3.1 in [19].

The following theorem gives the conditions for a point to be a local $(\eta, e)$-quasi efficient solution of (VOP) in terms of (MVVI).

Theorem 2. Suppose $-f$ is approximate $(\eta, e)$-invex at $\xi \in X$ such that $\eta(x, \xi)+\eta(\xi, x)=0$ for all $x \in X$. If $\xi$ is a solution of (MVVI), then $\xi$ is also a local $(\eta, e)$-quasi efficient solution of $(V O P)$.

Proof. Assume the vector $\xi$ fails to be a local $(\eta, e)$-quasi efficient solution of (VOP). Thus for each $r>0$ there is $x_{0} \in B(\xi, r)$ satisfying (3). Again the approximate $(\eta, e)$-invexity of $-f$ at $\xi$ yields

$$
(-f)(\xi)-(-f)\left(x_{0}\right) \geq_{C} A_{x_{0}} \eta\left(\xi, x_{0}\right)-e\left\|\eta\left(\xi, x_{0}\right)\right\|, \quad \forall A_{x_{0}} \in \partial(-f)\left(x_{0}\right) .
$$

Therefore

$$
f\left(x_{0}\right)-f(\xi) \geq_{C} A_{x_{0}} \eta\left(\xi, x_{0}\right)-e\left\|\eta\left(\xi, x_{0}\right)\right\|, \quad \forall A_{x_{0}} \in \partial(-f)\left(x_{0}\right) .
$$

Using (3) and taking into account the fact that $\partial(-f)\left(x_{0}\right)=-\partial f\left(x_{0}\right)$ and $\eta\left(x_{0}, \xi\right)=-\eta\left(\xi, x_{0}\right)$, we obtain

$$
\begin{aligned}
A_{x_{0}} \eta\left(x_{0}, \xi\right)=\left(-A_{x_{0}}\right) \eta\left(\xi, x_{0}\right) & \leq_{C} f\left(x_{0}\right)-f(\xi)+e\left\|\eta\left(\xi, x_{0}\right)\right\| \\
& =f\left(x_{0}\right)-f(\xi)+e\left\|\eta\left(x_{0}, \xi\right)\right\| \\
& \leq_{C} 0,
\end{aligned}
$$

for any $A_{x_{0}} \in \partial f\left(x_{0}\right)$. Hence $\xi$ does not solve (MVVI).

Furthermore, we prove that every solution of (SVVI) is still a local $(\eta, e)$-quasi efficient solution of (VOP) in the case of approximate pseudo invexity of type II.

Theorem 3. Assume that $f$ is approximate pseudo $(\eta, e)$-invex of type II at $\xi \in X$. If $\xi$ is a solution of (SVVI), then $\xi$ is also a local ( $\eta, e)$-quasi efficient solution of (VOP).

Proof. By contrapositive, assume that for each $r>0$, there is $x_{0} \in B(\xi, r)$ satisfying

$$
f\left(x_{0}\right)-f(\xi) \leq_{C}-e\left\|\eta\left(x_{0}, \xi\right)\right\|<_{C} 0 .
$$

Since $f$ is approximate pseudo $(\eta, e)$-invex of type II at $\xi$, it follows that

$$
A_{\xi} \eta\left(x_{0}, \xi\right)<_{C}-e\left\|\eta\left(x_{0}, \xi\right)\right\|, \quad \forall A_{\xi} \in \partial f(\xi) .
$$

Applying equation (4), we get

$$
A_{\xi} \eta\left(x_{0}, \xi\right) \leq_{C} 0, \quad \forall A_{\xi} \in \partial f(\xi) .
$$

Therefore $\xi$ is not a solution of (SVVI).

Using similar arguments as in the proof of Theorem (2), we can also demonstrate that under approximate pseudo $(\eta, e)$-invexity of type II of the function $-f$, solutions to (MVVI) are also local $(\eta, e)$-quasi efficient solutions of (VOP) provided that $\eta$ holds the same condition. 


\section{Sufficient and necessary conditions for local quasi weak efficient so- lutions}

In this section, we consider the weak formulations of Stampacchia [22] and Minty [17] vector variational inequalities as follows:

(WSVVI) Find $\xi \in X$ for which there exists no $x \in X$ satisfying

$$
A_{\xi} \eta(x, \xi)<_{C} 0, \quad \forall A_{\xi} \in \partial f(\xi)
$$

(WMVVI) Find $\xi \in X$ for which there exists no $x \in X$ satisfying

$$
A_{x} \eta(x, \xi)<_{C} 0, \quad \forall A_{x} \in \partial f(x)
$$

First, let us note that applying similar arguments as in the previous section, we can show that if $f$ is approximate pseudo $(\eta, e)$-invex of type I at a solution $\xi \in X$ of (WSVVI), then $\xi$ is also a local $(\eta, e)$-quasi weak efficient solution of (VOP). The converse implication is provided by the following theorem.

Theorem 4. Suppose that $\eta$ is affine in the first argument with $\eta(x, x)=0$ for all $x \in X$. Assume also that $-f$ is approximate quasi $(\eta, e)$-invex of type II at $\xi \in X$. If $\xi$ is a local $(\eta, e)$-quasi weak efficient solution of (VOP), then $\xi$ is a solution of (WSVVI).

Proof. Assume that $\xi$ is not a solution of (WSVVI). This means there is $x \in X$ such that for all $A_{\xi} \in \partial f(\xi)$, we have $A_{\xi} \eta(x, \xi)<_{C} 0$. Hence

$$
-A_{\xi} \eta(x, \xi)>_{C} 0
$$

From $\partial(-f)(x)=-\partial f(x)$ we get $-A_{\xi} \in \partial(-f)(\xi)$. Since $-f$ is approximate quasi $(\eta, e)$-invex of type II at $\xi$, there is $\widetilde{r}>0$ such that for any $x_{0} \in B(\xi, \widetilde{r})$

$$
\begin{aligned}
-A_{\xi} \eta\left(x_{0}, \xi\right)>_{C} 0 & \Rightarrow-f\left(x_{0}\right)-(-f(\xi))>_{C} e\left\|\eta\left(x_{0}, \xi\right)\right\| \\
& \Rightarrow f\left(x_{0}\right)-f(\xi)<_{C}-e\left\|\eta\left(x_{0}, \xi\right)\right\| .
\end{aligned}
$$

Let $r>0$ be arbitrary. We take $\bar{r} \leq \min \{r, \widetilde{r}\}$ and $\lambda \in(0,1)$ so that $x_{0}=\lambda x+(1-\lambda) \xi \in$ $B(\xi, \bar{r}) \subseteq X$. We have

$$
A_{\xi} \eta\left(x_{0}, \xi\right)=A_{\xi} \eta(\lambda x+(1-\lambda) \xi, \xi)=\lambda A_{\xi} \eta(x, \xi)
$$

thanks to the assumptions on $\eta$. Thus, by using (5), we obtain $-A_{\xi} \eta\left(x_{0}, \xi\right)>_{C} 0$. Since $x_{0} \in B(\xi, \widetilde{r})$, then applying $(6)$, the last inequality yields

$$
f\left(x_{0}\right)-f(\xi)<_{C}-e\left\|\eta\left(x_{0}, \xi\right)\right\|
$$

where $x_{0} \in B(\xi, r)$. Hence, $\xi$ cannot be an $(\eta, e)$-quasi weak efficient solution of (VOP).

The following theorem illustrates when a solution of (WMVVI) is also a local $(\eta, e)$-quasi weak efficient solution of (VOP).

Theorem 5. Suppose that $-f$ is approximate pseudo $(\eta, e)$-invex of type $I$ at $\xi$ with $\eta(x, \xi)+$ $\eta(\xi, x)=0$ for all $x \in X$. If $\xi$ is a solution of (WMVVI), then $\xi$ is a local $(\eta, e)$-quasi weak efficient solution of (VOP). 
Proof. Assume that for each $r>0$ there is $x_{0} \in B(\xi, r)$ satisfying

$$
f\left(x_{0}\right)-f(\xi)<_{C}-e\left\|\eta\left(x_{0}, \xi\right)\right\| .
$$

Since $\eta\left(x_{0}, \xi\right)=-\eta\left(\xi, x_{0}\right)$, we obtain

$$
-f(\xi)-(-f)\left(x_{0}\right)<_{C}-e\left\|\eta\left(\xi, x_{0}\right)\right\|
$$

As the function $-f$ is approximate pseudo $(\eta, e)$-invex of type $\mathrm{I}$ at $\xi$, it follows that

$$
A_{x_{0}} \eta\left(\xi, x_{0}\right)<_{C}-e\left\|\eta\left(\xi, x_{0}\right)\right\|, \quad \forall A_{x_{0}} \in \partial(-f)\left(x_{0}\right) .
$$

Using $\partial(-f)\left(x_{0}\right)=-\partial f\left(x_{0}\right)$ and $\eta\left(x_{0}, \xi\right)=-\eta\left(\xi, x_{0}\right)$, we obtain

$$
A_{x_{0}} \eta\left(x_{0}, \xi\right)=\left(-A_{x_{0}}\right) \eta\left(\xi, x_{0}\right)<_{C}-e\left\|\eta\left(\xi, x_{0}\right)\right\|<_{C} 0
$$

for any $A_{x_{0}} \in \partial f\left(x_{0}\right)$.

The next result specifies that vector critical points represent sufficient optimality conditions. Let us first recall their definition.

Definition 4. A vector critical point of $f$ is a feasible point $\xi \in X$ so that the system $\mu^{T} A_{\xi}=0$ admits a solution $\mu>_{C} 0$ for some $A_{\xi} \in \partial f(\xi)$ [15].

The gist of the above definition is that a vector critical point $\xi$ means $0 \in \mu^{T} \partial f(\xi)$ has at least a positive solution $\mu$. Note that in case of a scalar-valued objective function $f$, a critical point $\xi$ is a solution to the inclusion problem $0 \in \partial f(\xi)$.

Theorem 6. Assume $f$ is approximate pseudo $(\eta, e)$-invex of type I at $\xi \in X$. If $\xi$ is a vector critical point of $f$, then $\xi$ is a local $(\eta, e)$-quasi weak efficient solution of (VOP).

To prove this result, we need to use the following theorem of the alternative.

Lemma 1. (Gordan's Theorem) [6] If $A$ is a $n \times m$ matrix, then we have either

1. Ax $<_{C} 0$ for some $x \in \mathbb{R}^{m}$; or

2. $A^{T} y=0, y \geq_{C} 0 \quad$ for some nonzero solution $y \in \mathbb{R}^{n}$;

but not both.

Proof of Theorem 6. Assume that for each $r>0$, there is $x_{0} \in B(\xi, r)$ satisfying

$$
f\left(x_{0}\right)-f(\xi)<-e\left\|\eta\left(x_{0}, \xi\right)\right\| .
$$

Since $f$ is approximate pseudo $(\eta, e)$-invex of type $\mathrm{I}$ at $\xi$, we obtain

$$
A_{\xi} \eta\left(x_{0}, \xi\right)<_{C} 0, \quad \forall A_{\xi} \in \partial f(\xi) .
$$

By applying Gordan's Theorem we deduce that there is no $\mu>_{C} 0$ such that $\mu^{T} A_{\xi}=0$ for all $A_{\xi} \in \partial f(\xi)$. We conclude $\xi$ is not a vector critical point of $f$.

Remark 3. Theorem 6 improves Lemma 3.1 in [19] since the approximate pseudo convexity of type I has been weakened by the approximate pseudo $(\eta, e)$-invexity of type I. 


\section{Example}

In this section, we illustrate the obtained results by an example.

Consider the following vector optimization problem:

$$
\min f(x):=\left(f_{1}(x), f_{2}(x)\right), \quad \text { s.t. } \quad x \in X
$$

where,

$$
f_{1}(x)= \begin{cases}-x^{3}-x^{2}+5 x & x \geq 0 \\ x^{3}+6 x & x<0\end{cases}
$$

and

$$
f_{2}(x)= \begin{cases}x^{2}-2 x & x \geq 0 \\ -x^{2}-3 x & x<0\end{cases}
$$

$X=\mathbb{R}, C=\mathbb{R}_{+}^{2}$ and $\eta(x, y)=x-y$ for all $x, y \in X$.

The Clarke subdifferential of $f$ at $x \in X$ is given by

$$
\partial f(x)= \begin{cases}\left\{\left(-3 x^{2}-2 x+5,2 x-2\right)^{T}\right\} & x>0 \\ c o\left\{(5 ;-2)^{T},(6 ;-3)^{T}\right\}=\left\{\left(5 k_{1}+6 k_{2} ;-2 k_{1}-3 k_{2}\right)^{T}\right\} & x=0 \\ \left\{\left(3 x^{2}+6,-2 x-3\right)^{T}\right\} & x<0\end{cases}
$$

where $k_{1} \geq 0, k_{2} \geq 0$ such that $k_{1}+k_{2}=1$.

For any $e=\left(e_{1}, e_{2}\right)$ s.t $0<e_{i}<1$ with $i \in\{1,2\}$, we prove that there exists $r=\frac{1}{2}>0$ such that, for all $x, y \in B\left(x_{0}, r\right), x_{0}=0$, one has

$$
f(x)-f(y)<_{C} 0 \quad \Rightarrow \quad A_{y} \eta(x, y)+e\|\eta(x, y)\|<_{C} 0, \forall A_{y} \in \partial f(y),
$$

Hence, $f$ is approximate pseudo $(\eta, e)$-invex of type II at $x_{0}$.

On the other hand, let $\xi=0$.

Since for any $x \in X \backslash\{\xi\}$ and for all $k_{1} \geq 0, k_{2} \geq 0$ such that $k_{1}+k_{2}=1$ one has

$$
A_{\xi} \eta(x, \xi)=x\left(5 k_{1}+6 k_{2} ;-2 k_{1}-3 k_{2}\right)^{T} \not_{C} 0,
$$

Therefore, $\xi=0$ solves (SVVI).

Now, since $f$ is approximate pseudo $(\eta, e)$-invex of type II at $\xi$, then, by Theorem $3, \xi=0$ should be a local $(\eta, e)$-quasi efficient solution of (VOP). Indeed, for $0<e<1$, we have for all $x \in B(\xi, r) \backslash\{\xi\}$ with $r>0$

$$
f(x)-f(\xi)+e\|\eta(x, \xi)\|=f(x)+e|x|=\left\{\begin{array}{ll}
\left(-x^{3}-x^{2}+5 x+e x, x^{2}-2 x+e x\right)^{T} & x>0 \\
\left(x^{3}+6 x-e x,-x^{2}-3 x-e x\right)^{T} & x<0
\end{array},\right.
$$

which means that

$$
f(x)-f(\xi)+e\|\eta(x, \xi)\| \not_{C} 0,
$$

Therefore, $\xi$ is a local $(\eta, e)$-quasi efficient solution of (VOP). 


\section{Conclusion}

We have considered two generalized types of quasi efficient solutions to nonsmooth vector optimization problems. Using new generalized invexity assumptions we have provided necessary and sufficient conditions of optimality for these solutions. More precisely, we have shown that Stampacchia vector variational inequalities present sufficient optimality conditions when the multiobjective function $f$ satisfies weak forms of approximate invexity. On the other hand, we have obtained necessary optimality conditions in terms of Minty vector variational inequalities given similar approximate invexity assumptions on the function $-f$. Finally, we have proven that vector critical points represent sufficient optimality conditions as well for approximate pseudo invex functions. It is worth mentioning that we have used the original definition of Clarke's generalized Jacobian instead of the Cartesian product of Clarke's subdifferentials of the scalarvalued function components as done in $[14,15,18,19]$. Since our generalized invexity contains as special cases previous generalized convexity conditions that were provided in $[7,5,20]$, the presented theorems in this paper extend many corresponding results in the literature like [19] for example.

\section{References}

[1] Al-Homidan, S. and Ansari, Q. H. (2010). Generalized minty vector variational-like inequalities and vector optimization problems. Journal of Optimization Theory and Applications, 144(1), 1-11. doi: 10.1007/s10957-009-9591-7

[2] Ansari, Q. H., Köbis, E. and Yao, J. C. (2018). Vector variational inequalities and vector optimization. Cham: Springer. doi: 10.1007/978-3-319-63049-6

[3] Ansari, Q. H. and Rezaei, M. (2013). Generalized vector variational-like inequalities and vector optimization in Asplund spaces. Optimization - A Journal of Mathematical Programming and Operations Research, 62(6), 721-734. doi: 10.1080/02331934.2012.669758

[4] Aslam Noor M., Mishra, S. K. and Momani, S. (2005). Properties of approximate preinvex functions. Nonlinear Analysis Forum, 10(2), 227-235. http://prof .ks.ac.kr/bslee/naf/table/ vol-1002/10-NAF-1002.pdf

[5] Aslam Noor, M. and Inayat Noor, K. (2006). Some characterizations of strongly preinvex functions. Journal of Mathematical Analysis and Applications, 316(2), 697-706. doi: 10.1016/j.jmaa.2005.05.014

[6] Bazaraa, M. S., Sherali, H. D. and Shetty, C. M. (2006). Nonlinear programming: Theory and algorithms. New York: Wiley. doi: 10.1002/0471787779

[7] Bhatia, D., Gupta, A. and Arora, P. (2013). Optimality via generalized approximate convexity and quasiefficiency. Optimization Letters, 7, 127-135. doi: 10.1007/s11590-011-0402-3

[8] Clarke, F. H. (1983). Optimization and nonsmooth analysis. New York: Wiley. doi: $10.1137 / 1.9781611971309$

[9] Eichfelder, G. and Jahn J. (2012). Vector optimization problems and their solution concepts. In Hasan Ansari Q. and Yao, J.-C. (Eds.), Recent Developments in Vector Optimization (pp. 1-27). Berlin: Springer. doi: 10.1007/978-3-642-21114-0_1

[10] Fang, Y. P. and Hu, R. (2009). A nonsmooth version of Minty variational principle. Optimization, 58(4), 401-412. doi: 10.1080/02331930701762845

[11] Gang, X. and Liu, S. (2008). On Minty vector variational-like inequality. Computer and Mathematics with Applications, 56(2), 311-323. doi: 10.1016/j.camwa.2007.12.011

[12] Giannessi, F. (1980). Theorems of the alternative: quadratic programs and complementarity problems. In Cottle, R. W., Giannessi, F. and Lions, J.-L. (Eds.), Variational Inequalities and Complementarity Problems - Theory and Applications (pp. 151-186). New York: Wiley.

[13] Giannessi, F. (1998). On Minty variational principle. In Giannessi, F., Komlósi, S. and Rapcsák, T. (Eds.), New Trends in Mathematical Programming (pp. 93-99). Dordrecht: Springer. doi: 10.1007/978-1-4757-2878-1_8 
[14] Gupta, P. and Mishra, S. K. (2018). On Minty variational principle for nonsmooth vector optimization problems with generalized approximate convexity. Optimization - A Journal of Mathematical Programming and Operations Research, 67(8), 1157-1167. doi: 10.1080/02331934.2018.1466884

[15] Gutiérrez, C., Jiménez, B., Novo, V. and Ruiz-Garzón, G. (2016). Vector critical points and efficiency in vector optimization with Lipschitz functions. Optimization Letters, 10, 47-62. doi: 10.1007/s11590-015-0850-2

[16] Long, X. J., Peng, J. W. and Wu, S. Y. (2012). Generalized vector variational-like inequalities and nonsmooth vector optimization problems. Optimization - A Journal of Mathematical Programming and Operations Research, 61(9), 1075-1086. doi: 10.1080/02331934.2010.538056

[17] Minty, G. J. (1967). On the generalization of a direct method of the calculus of variations. Bulletin of the American Mathematical Society, 73(3), 315-321. doi: 10.1090/s0002-9904-1967-11732-4

[18] Mishra, S. K. and Wang, S. Y. (2006). Vector variational-like inequalities and non-smooth vector optimization problems. Nonlinear Analysis: Theory, Methods and Applications, 64(9), 1939-1945. doi: 10.1016/j.na.2005.07.030

[19] Mishra, S. K. and Upadhyay, B. B. (2013). Some relations between vector variational inequality problems and nonsmooth vector optimization problems using quasi efficiency. Positivity, 17(4), 1071-1083. doi: 10.1007/s11117-013-0221-0

[20] van Ngai, H., Luc, D. and hera, M. (2000). Approximate convex functions. Journal of Nonlinear and Convex Analysis, 1(2), 155-176. http://www.ybook.co.jp/online2/jncav1.html

[21] Oveisiha, M. and Zafarani, J. (2012). Vector optimization problem and generalized convexity. Journal of Global Optimization, 52(1), 29-43. doi: 10.1007/s10898-011-9657-6

[22] Stampacchia, G. (1960). Formes bilinéaires coercitives sur les ensembles convexes. Comptes Rendus Académie des Sciences de Paris, 258, 4413-4416.

[23] Yang, X. M., Yang, X. Q. and Teo, K. L. (2004). Some remarks on the Minty vector variational inequality. Journal of Optimization Theory and Applications, 121(1), 193-201. doi: 10.1023/B:JOTA.0000026137.18526.7a

[24] Yang, X. M. and Yang, X. Q. (2006). Vector variational-like inequalities with pseudoinvexity. Optimization - A Journal of Mathematical Programming and Operations Research, 55(1-2), 157170. doi: 10.1080/02331930500530609 